\title{
SOME ASPECTS OF LOCAL ANAESTHESIA
}

\author{
By R. P. HARBORD, M.D., D.A., R.A.M.C.
}

Satisfactory as local anaesthesia can be, when the anaesthetic limits are trespassed by the operator, the distress produced may have more far-reaching effects than is generally supposed.

Success dẹpends as much upon a knowledge of the regional nerve supply as upon an appreciation of the extent to which it can be effectively blocked. A spinal subarachnoid block up to the level of the fourth or fifth dorsal segment does not adequately protect the stomach from traumatic impulses, for although the splanchnic nerves are given off below this the vagi remain intact. While these may be blocked in this area by infiltrating the anterior and posterior parts of the oesophagus with novocain, it may be that other organs in the peritoneal cavity require to be dealt with. If the operator is capable of such gentle handling as not to cause vagal distress, then much can be achieved inside the abdomen with local methods. It may be that this is the only possible way for a successful issue. Broadly speaking, local analgesia is not a good method for operations on the abdominal viscera in which much trauma is anticipated. When adequate control is not possible, then general anaesthesia is indicated. There are circumstances when the pathology is such that a certain amount of handling of the tissues is necessary which could hardly be described as gentle. It is in these cases that local analgesia fails.

Premedication allays nervous apprehension and may even result in amnesia. Omnopon grains $2 / 3$ rds, and scopolamine, grains I/I5oth, serves in the case of healthy adults of over twelve stones weight; omnopon, grains $I / 3$ rd, and scopolamine, grains $I / I 50 t h$, for those between this and seven stones; while for adult patients under this the doses are reduced to $I / 6$ th and $\mathrm{I} / 300$ th grain respectively (and in the case of children morphine is given by body weight, $\mathrm{r} / 4$ oth grain per stone). If little or no drowsiness results a further injection of omnopon may be given intravenously. The administration must be made slowly and in fractional doses of $\mathrm{I} / 6$ th grain till the required effect is obtained.

Basal narcosis with local analgesia is unsatisfactory as it is unreliable, and may result in a state in which the patient becomes unmanageable, moving about, and possibly requiring restraint, with danger to the sterile field.

Amnesia does not mean that there has not been any mental shock, nor does it signify that nocciceptive impulses have been blocked. It is not uncommon, however, for a patient to ask when his operation is to be done after it has actually been accomplished. I have on record the case of a man, aged forty-five years, who had a palliative gastroenterostomy for a carcinoma. He was small in stature and pale, but there was no apparent cachexia. After omnopon and scopolamine he was drowsy, but not asleep, and a field block of the abdominal wall was carried out. The peritoneum was also infiltrated with novocain, but this was all. Nothing untoward occurred except at critical phases, such as when the stomach was delivered out of the peritoneal cavity, and towards the end of the operation when the viscera were returned before suture of the abdominal wall. At these times the patient groaned out loud, and there was some distress. There was a moderate but temporary fall in blood pressure. Two days later he complained bitterly that he had been in hospital long enough, and that it was time his operation was done. Three days later he died after a period in which the pulse rate kept rising, but nothing else abnormal was detected. There was no autopsy, but the impression at the time was that the myocardium had failed.

In this type of fatality, where the diagnosis is uncertain, the question of internal haemorrhage, or extravasion of blood under cover of a serous membrane, must be considered. In the case described, the abdomen was soft and not distended, and there was no increase in pallor.

An examination of this case reveals two points:-

I. Impulses must have reached the cerebrum as shown by the response during the critical phases of the operation.

2. The state of amnesia does not preclude the possibility of shock.

Was there a psychogenic factor responsible? If so, what was the degree of its effect proportionately?

The effects of nervous exhaustion shock, from unblocked impulses due to trauma, and 
affecting the bodily system as a whole, vary according to whether there is debility or otherwise. Any normal person can withstand a certain amount of sustained trauma, but there is a breaking point. Primary shock tends to limit the effects by producing unconsciousness. It is the normal reaction. In the patient debilitated by disease, or in one who has a low blood pressure, the onset of shock will be more rapid and the effects more severe. It is impossible to assess this factor in relation to the average type, but some idea of it can be obtained in the individual case, and each should be judged on its own merits. It is highly probable that psychogenic disturbance may be followed by endocrine dysfunction, and this may, in turn, have its effects upon the systems of the body. After awakening from a disturbing dream a normal patient feels exhausted, although he may remember little or nothing of it. In the case described above it is therefore possible that the myocardial failure may have been the result of such a factor.

There is much to be said, therefore, for the principals of "anoci-association" laid down by Crile in I9I3, in which he advocated morphine for mental apprehension, local anaesthesia to prevent the origin of traumatic impulses, and general anaesthesia to protect the cerebrum.

When general anaesthesia is given with local analgesia, a light plane is all that is necessary, since many of the impulses will be blocked. The depth should be sufficient to prevent the patient from moving his arms or legs. According to Guedel's classification the first plane of the third stage would be adequate, but care must be exercised to see that the second. stage does not appear otherwise the patient may vomit or move. Where an endotracheal tube is in situ this should have been lubricated with a ro per cent percaine paste, so that the plane of anaesthesia may remain light without producing paroxysms of coughing. Perhaps the easiest anaesthesia to control is cyclopropane-oxygen with carbon dioxide absorption, on account of the rapidity with which the depth can be adjusted.

An intravenous barbiturate has the advantage that it has to some extent a counteracting influence on the noxious effects of novocain. It may be combined with cyclopropane-oxygen. If the barbiturate is used with local anaesthesia alone there may come a time when the effects of the former will wear off and the patient may become unmanageable. This is not the case, however, when the barbiturate is given continuously. Inhalational anaesthesia in the hands of an expert is preferable, since it is more readily controllable, especially where the operation is prolonged.

Some of the pharmacological effects of analgesic drugs are worthy of note. Novocain injections in quantity dilate the vessels of the skin, particularly of the face. The flushed facies may actually conceal fall in blood pressure, although this may readily be demonstrated by noting the change in the radial or superficial temporal pulse.

A sudden collapse may occur if novocain is injected intravenously, even when no adrenalin has been present. The characteristic feature of this in animals, and sometimes in man, is cessation of respiration, and there may be convulsions. Artificial respiration is imperative, the best method being by repeated manual compression of a bag filled with oxygen and applied to the face by a tightly fitting mask. If available carbon dioxide may be added in 5 to ro per cent by volume. The mechanism of this type of collapse is not altogether clear, since large amounts of novocain have been introduced experimentally by the blood stream with no ill-effects. It should be noted in passing that carbon dioxide should not be given in collapse due to spinal shock, as this gas may on occasion cause a further fall in blood pressure, as there is evidence that it dilates the peripheral vessels.

Local analgesia alone is indicated, especially where the whole area of operation field can be blocked. A drop of 4 per cent cocaine instilled into the conjunctival sac is sufficient for eye operations. A ro per cent spray with adrenalin, i.e. equal volumes of 20 per cent cocaine and I in I,000 adrenalin, produces good analgesia for intranasal operations. The danger of cocaine idiosyncrasy in children is a practical one. Surface analgesia is also effective in the urethra. The urethra may also be rendered analgesic by means of a low spinal $(0.4 \mathrm{~cm}$. stovaine given in the "sitting bull position" for three minutes), or by the injection of $30 \mathrm{cms}$. of I per cent novocain into the sacral canal.

For operations on the bladder and prostate a spinal block up to the umbilicus is sufficient as these are extra-peritoneal structures. A spinal anaesthetic is not an efficient block for lower abdominal work for several reasons, one being that it does not block the vagus, and another that the organs of the upper abdomen may require examination.

For partial gastrectomy a local field block of the abdominal wall combined with infiltration of the mesenteries of the stomach and cyclopropane-oxygen gives good results. The object of 
infiltrating the mesenteries is to block the sympathetic nerves, and this is more advantageous than a splanchnic block on account of there being little or no fall in blood pressure with the former. A local field block of the abdominal wall combined with relatively light general anaesthesia is a good combination for gall bladder surgery.

Operations on the ureters and kidneys are extra-peritoneal and can be performed with spinal anaesthesia, preferably with the hypobaric solutions.

Caesarian section is an extra peritoneal operation when performed at the lower segment, but it is not wise to employ a spinal anaesthetic as there has been a definite mortality associated with this. Local analgesia can be used as the sole anaesthetic, but a light general is in my opinion preferable.

While many hernial operations can be performed satisfactorily with spinal analgesia some local anaesthetic can with advantage be infiltrated in the neighbourhood of the neck of the sac.

Operations on the lower limbs may be performed with spinal analgesia which produces a complete block.

Brachial plexus block may produce a marked fall in blood pressure. Operations on the neck, e.g. removal of a non-toxic adenoma, can be performed with a cervical plexus block. Great care is necessary here owing to the proximity of the large vessels in order that the solution is not injected intravenously.

Intercostal block for empyema may be rendered complete if the intercostal nerves are injected throughout their course in the operation path. This ensures the completeness of the nerve block.

The advantages of local analgesia in brain surgery are (I) less haemorrhage, (2) less tendency to produce coughing and straining, (3) there is no rise in the intracranial tension. The brain itself is insensitive.

While most operations can be completed with local analgesia alone, its particular value is when inhalational anaesthesia is contraindicated as in certain acute respiratory conditions.

In cases of renal failure care should be taken to reduce the total dose of local anaesthetics and to use the weakest strengths, e.g. $\frac{1}{4}$ novocain in order not to affect the blood pressure.

The drugs most frequently used are novocain, percaine, and novutox. Stovaine is being used less frequently than formerly. Cocaine is only used for surface analgesia.

Novocain is given by infiltration in strengths of $\frac{1}{4}$ to 2 per cent, and the total dose should not exceed I gramme when using the I per cent solution. The stronger the concentration the smaller should be the total dose, as it appears that the concentrated solution can be absorbed the most rapidly. It is more usual to use the $\frac{1}{4}$ per cent strength when a greater quantity has to be used. Adrenalin may be added in the ratio of I minim of I in I,000 solution to 30 c.c., in order to limit both the absorption of the drug, and the bleeding, by producing a vasoconstriction of the local vessels.

A few sudden fatalities from the accidental intravenous injection have taken place. It is therefore important to withdraw the plunger of the syringe prior to injection.

Percaine $I$ in $I, 500$ produces an analgesia which may last twice as long as novocain, that is about two hours. 300 c.c. is the maximum dose.

Novutox has novocain as the basis and remains sterile even after exposure to the air.

Servocaine is said to have a rapid initial effect and to last as long as percaine.

Solutions of cocaine and adrenalin should be freshly prepared, otherwise decomposition takes place.

A continuous flow syringe of the Rowbotham or Sana-Lok-Pitkin type will be found advantageous where large quantities are given. Syringes should not be boiled with alkalis as these tend to precipitate the drugs. They should be boiled and then immersed in spirit or sterile water before use. Novocain and percaine may be sterilised by boiling.

The main contraindications to local analgesia are sepsis of the tissues concerned and nervous conditions. The local anaesthetic may be infiltrated away from the infected area as, for example, in the case of a block of the maxillary nerve for the removal of septic teeth. It must be remembered that this procedure will bring about vascular changes in the area concerned, and that this may have some effect on the healing of the tissues. This is a doubtful point, but is worthy of note.

Children make poor patients for local analgesia, but in infants the operation of dividing the hypertrophied muscle of the pylorus in congenital pyloric stenosis has been performed with some success with local analgesia and morphia. 
Those with psychoneuroses or psychoses are better treated with general anaesthesia as well as normal people of nervous disposition, or those who have a rooted objection to "being present at their own operation."

\title{
Conclusion.
}

While it is true that local analgesia may be used for most operations, many are best performed in conjunction with inhalational anaesthesia with adequate premedication.

The psychological factors where local analgesia is relatively inadequate may be neglected in the healthy adult but should not be overlooked in the debilitated and may in these cases result in systemic disorders persisting and perhaps contributing to a fatal conclusion.

\section{CORRESPONDENCE}

\author{
Medical Planning \\ Evolution or Revolution?
}

SiR,

The present House of Commons appears rather to have lost its head! In a way this is understandable. The nation has consented to the exercise of such wide powers for the purpose of the successful prosecution of the war that a virtual dictatorship in that respect has been conceded.

In relation to the proposed medical legislation it seems strange that the Law Officers of the Crown have not gently tapped Mr. Ernest Brown on the shoulder and said, "Can't you read the notice, 'PRIVATE. Entrance by permission only'!" No permission has been granted by the nation for any revolutionary legislation of a non-urgent nature whatever. This Government has no right at all to take any action which is a clear infringement of the Constitution. If the claim is made that the proposal is not revolutionary, but is justifiable because it is designed for the good of the nation, is that not the first stage of the totalitarian outlook whereby the future of the community is planned by a relatively small group of men wichout reference to the wishes of that community! This matter affects a complete profession of some 70,000 men and women, and practically the whole of the population of these islands, not just for the period of the war, but throughout the long days of peace which we trust will follow. Is this such a trivial question that it can be dealt with by a Parliament elected years ago, and only still allowed to scrve as a matter of wartime expediency? In the spate of emergency orders (which we intend to see swept away at the earliest possible moment after the war, together with a host of redundant civil servants) our politicians seem entirely to have forgotten the whole of the British national conception of progress in ordinary peaceful development. They would be well advised to study the single letter " $R$ " in the subtitle of these remarks!

As a nation we prefer instinctively to evolve slowly, taking out a few bricks from the edifice here, putting others in their place, standing back to survey the effect, and continuing the process by easy and reasoned stages. We do not believe in wholesale demolitions and rapid replacement by pretentious jerry-building. We do not approve of sudden and drastic re-organisation like that proposed for the medical profession.

Most people will admit that the present structure has serious faults, but we have a sure enough foundation on which to build. That is the time-honoured relationship between practitioner and patient.

- A rough pencil-sketch of the first wise steps in altering the present system might be:-

(I) Fewer insured patients should be allowed per doctor, who would accordingly have time to give them better attention.

(2) An increased capitation fee to compensate for this.

(3) Consultant and specialist advice to be obtainable for all insured persons by the agreement of reasonable fees to be paid out of the accumulating millions in the coffers of the friendly societies.

(4) Hospital facilities to be available for all, in the case of the insured person by contributions from both State and Friendly Societies. Non-insured persons should be allowed treatment if they make reasonable personal contributions, the balance of expense being made up from the Exchequer.

(5) The profession should agree to take periodic post-graduate courses.

(6) A national pool of locum-tenens might be formed. Substitutes readily available would allow every practitioner to obtain that annual period of rest, free from worry, which is essential to his physical well-being and efficient performance of his professional duties.

(7) The Insurance scheme might be extended by agreement to other classes and their dependants.

(8) The money to meet the major portion of these proposals is really already available. The Friendly. Societies should be made to keep a separate Medical Benefit and Sickness Benefit account. The millions accumulated by the latter should be automatically available for transfer to the Medical Benefit account for the next year, allowing perhaps a 5 or ro per cent deduction for a Sickness Benefit reserve.

The above is not intended to be in any sense a complete scheme. It is merely an indication of how, without undue disturbance, the attempt at real progress might be made. 